\title{
The hyphenation of production-scale free-flow electrophoresis to electrospray ionization mass spectrometry using a highly conduc- tive background electrolyte
}

\author{
Sven Kochmann ${ }^{\mathrm{a}}$, Fletcher J. Agostino ${ }^{\mathrm{a}}$, Yves J. C. LeBlanc ${ }^{\mathrm{b}}$, Sergey N. Krylov ${ }^{\mathrm{a}}$ \\ ${ }^{\mathrm{a}}$ Department of Chemistry and Centre for Research on Biomolecular Interactions, York University, Toronto, Ontario \\ M3J 1P3, Canada \\ bCIEX, 71 Four Valley Drive, Vaughan, Ontario L4K 4V8, Canada
}

\section{Supporting information}

\section{TABLE OF CONTENTS}

- Reagents, FFE-MS setup, experimental parameters, and experimental conditions for mass spectrometry (S2)

- Calculating the conductivity of the electrolyte (S1)

- Calculating the potentials and currents of the electric circuits (S3)

- Calculating of the descriptors $H_{\text {asym }}$ and $H_{\text {leak }}$ (S7)

- Effect of grounding on analyte signals with new ESI-probe configuration (S10)

- Examining the influence of FFE on ESI-spray with new ESI-probe configuration (S10)

- Previous $\mu$ FFE-MS works (S11)

Note: In the following text, $\mathrm{P}_{\mathrm{x}}$ (roman $\mathrm{P}$ ) refers to the node/point in the electric circuit, while $P_{\mathrm{x}}$ (italic $P$ ) refers to the electrical potential in the corresponding point. The values of potentials are with respect to ground.

Safety considerations. The performed experiments involve high voltage sources, which interfere. This can lead to unpredictable effects and should, therefore, be performed with caution (danger of electrical shock). Therefore, appropriate precautions (e.g. protective gear, presence of an assistant) should be taken. 


\section{REAGENTS}

All solutions were prepared using analytical grade reagents. 4-(2-hydroxyethyl)piperazine-1-ethanesulfonic acid sodium salt (HEPES), rhodamine 6G hydrochloride (rhodamine), fluorescein sodium salt (fluorescein), and sodium hydroxide were purchased from Sigma Aldrich (Oakville, ON, Canada). Deionized water $\left(18.2 \mathrm{M} \Omega \mathrm{cm}^{-1}\right.$ ) was used for preparation of all solutions.

\section{FFE-MS SETUP}

A schematic of our FFE-MS setup is depicted in Figure S1. The setup consists of an FFE-device, in which the sample outlet 2 is directed towards the electrospray ionization (ESI) interface of the MS instrument by a transfer line (polyethylene tubing; inner diameter of 1/16”; $38 \mathrm{~cm}$ long). The effluent of outlets 1 and 3 are collected. Transfer line 2 and collection lines 1 and 3 (same material, diameter, and length as transfer line) are grounded by small metallic connectors (1 cm long) between the two pieces of tubing. The metallic connectors were connected to the case of the MS instrument, which is grounded. The lengths from the FFE outlets to the metal connectors were $24 \mathrm{~cm}$ each. The length from the metal connector of the transfer line to the MS was $14 \mathrm{~cm}$.

\section{EXPERIMENTAL PARAMETERS}

We used HEPES (10 mM, pH 7.5) as BGE and a mixture of rhodamine and fluorescein $(100 \mu \mathrm{M}$ each) as a well-studied model pair of analytes. Additionally, the individual dyes are visible to the naked eye, which allows their easy observation in FFE. Flow rates of the BGE and the sample solution were $5 \mathrm{~mL} \mathrm{~min}^{-1}$ and $5 \mu \mathrm{L} \mathrm{m^{-1 }}$, respectively. Liquid level of the chimneys was controlled by adjusting the flow rates at the electrolyte outlets by means of clamps (photo in Figure S1). The flush outlet at the far end of the chip was used only for purging the chip but was closed for the experiments. For purging, a $10 \%$ ethanol solution was passed through the device to wet the entire surface prior to the introduction of BGE. The FFE chip was cooled by a precooled metal block $\left(-20^{\circ} \mathrm{C}\right.$, $10 \times 8 \times 4 \mathrm{~cm}$ ), which was periodically exchanged (every 20-30 min).

\section{EXPERIMENTAL CONDITIONS FOR MS}

An AB Sciex API 5000 equipped with Turbo V TM source (Vaughan, ON, Canada) was used for the experiments. For the initial experiments, we used a standard stainless steel probe/electrode configuration. For later experiments (see Results and Discussion section in the main text) we used a fused silica probe (wide bore, $0.5 \mathrm{~mm}$ inner diameter) plumbed from the FFE coupling line all the way to the end of the probe. The MS instrument was calibrated before the first experiments and optimized for detection of the two analytes: fluorescein and rhodamine. Experimental conditions for MS measurements are listed in Table S1.

Table S1. Parameters of MS measurements.

\begin{tabular}{|l|l|}
\hline Parameter & Value(s) \\
\hline Vacuum Gauge & $3.3 \times 10^{-5}$ Torr (4 mPa) \\
\hline Polarity & Positive \\
\hline Collision gas & 6 psi (413 hPa) \\
\hline Curtain Gas ${ }^{\text {TM }}$ supply & 10 psi (689 hPa) \\
\hline Nebulizer gas (GS1) & 55 psi (3792 hPa) \\
\hline Heater gas (GS2) & 15 psi (1034 hPa) \\
\hline Ionspray voltage & $5500 \mathrm{~V}$ \\
\hline Source temperature & $100{ }^{\circ} \mathrm{C}$ \\
\hline Collision cell exit potential & $15 \mathrm{~V}$ \\
\hline Declustering potential & $50 \mathrm{~V}$ \\
\hline Mass ranges & $\mathbf{Q 1}(\mathbf{m} / \mathbf{z}), \mathbf{Q 3}$ (m/z), Dwell \\
\hline Fluorescein & $333.1,287.0,25.0$ \\
\hline & $333.1,271.0,25.0$ \\
\hline & $333.1,231.1,25.0$ \\
\hline Rhodamine & $444.2,416.0,25.0$ \\
\hline & $444.2,387.0,25.0$ \\
\hline & $444.2,327.0,25.0$ \\
\hline
\end{tabular}
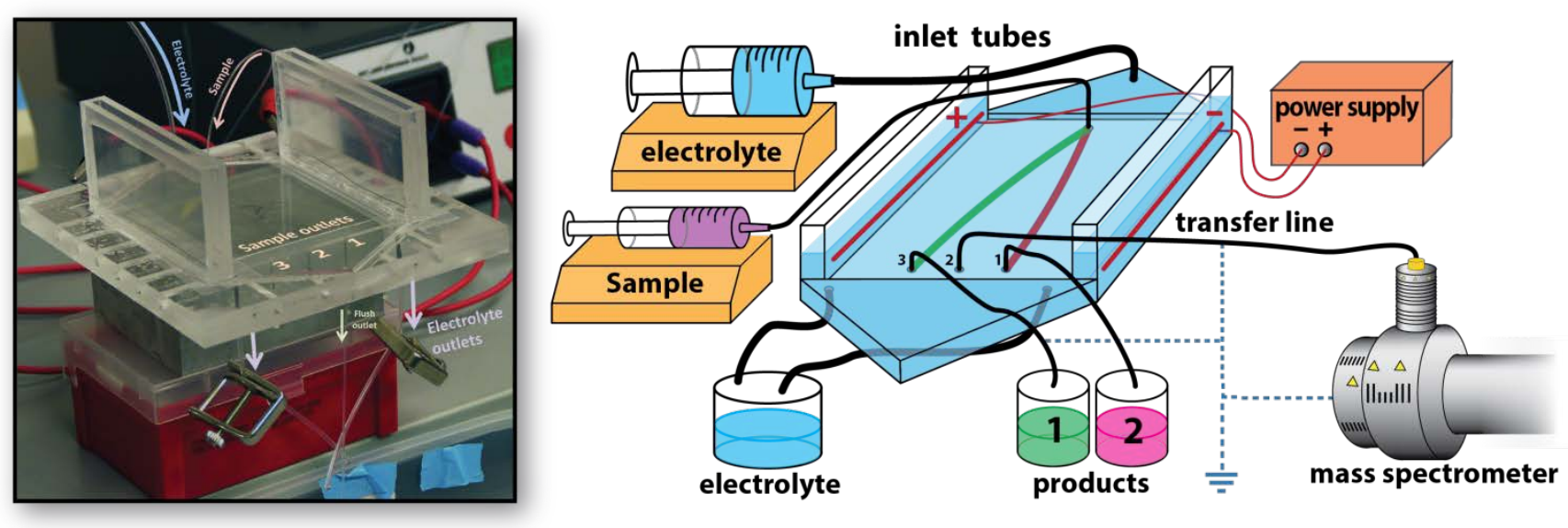

Figure S1. Photo and schematic of the FFE-MS setup. For clarity, only sample outlets 1 to 3 are depicted. 


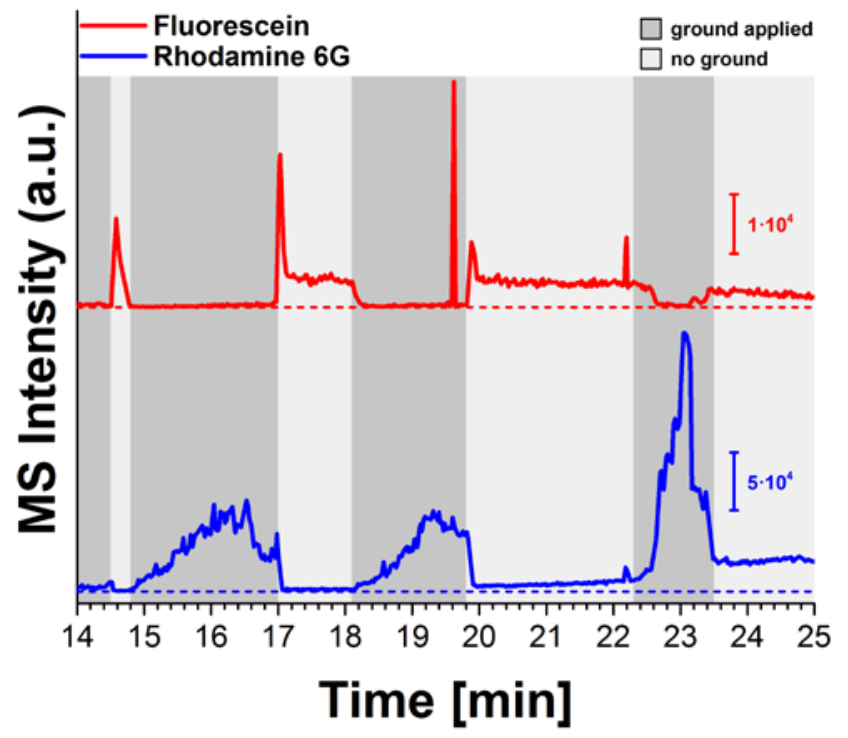

Figure S2. Influence of grounding state on fluorescein and rhodamine signals. The dashed lines represent the corresponding zero lines (baselines). For this experiment, no separation voltage was applied $\left(V_{\mathrm{FFE}}=0\right)$.

\section{CALCULATING THE CONDUCTIVITY OF THE ELECTROLYTE}

The electrical conductivity $\kappa$ of the background electrolyte (BGE) can be calculated as:

$\kappa=\frac{1}{\rho}=\frac{1}{R} \frac{L}{A}$

where $\rho$ is the electrical resistivity, $R$ is the electrical resistance between the two electrodes of the free flow electrophoresis (FFE) chip. The separation zone of the chip is defined by the cross-section area $A$ and the distance $L$ between the two electrodes. In our case, the cross-section area was $A=9 \mathrm{~cm}$ $\times 0.2 \mathrm{~cm}=1.8 \mathrm{~cm}^{2}$ and the distance between the electrodes was $L=10 \mathrm{~cm}$. For our measurements, we found that for an applied voltage of $750 \mathrm{~V}$, the average current $I_{\text {avg }}$ was $23.0 \mathrm{~mA}$ resulting in a resistance $R=33 \mathrm{k} \Omega$. The electrical resistivity and conductivity were $\rho=5.9 \Omega \mathrm{m}$ and $\kappa=1.7 \mathrm{mS} \mathrm{cm}^{-1}$, respectively.

\section{CALCULATING THE POTENTIALS AND CURRENTS OF THE ELECTRIC CIRCUITS}

Resistors. $\mathrm{P}_{2}$ can refer to the physical point of any outlet. Thus, the resistances $R_{1}$ and $R_{2}$ depend on the location of $\mathrm{P}_{2}$. Using Equation (S1), it is possible to express the ratio of the resistances $R_{1}$ and $R_{2}$ as a simple ratio $r_{\mathrm{L}}$ of lengths since the conductivity $\kappa$ and the cross-section area $A$ is the same for both resistors:

$$
\frac{R_{1}}{R_{2}}=\frac{L_{1}}{L_{2}}=r_{\mathrm{L}}
$$

where $L_{1}$ is the distance between $\mathrm{P}_{1}$ and $\mathrm{P}_{2}$ and $L_{2}$ is the distances between $\mathrm{P}_{2}$ and $\mathrm{P}_{3}$. Obviously, if $\mathrm{P}_{2}$ is exactly in the
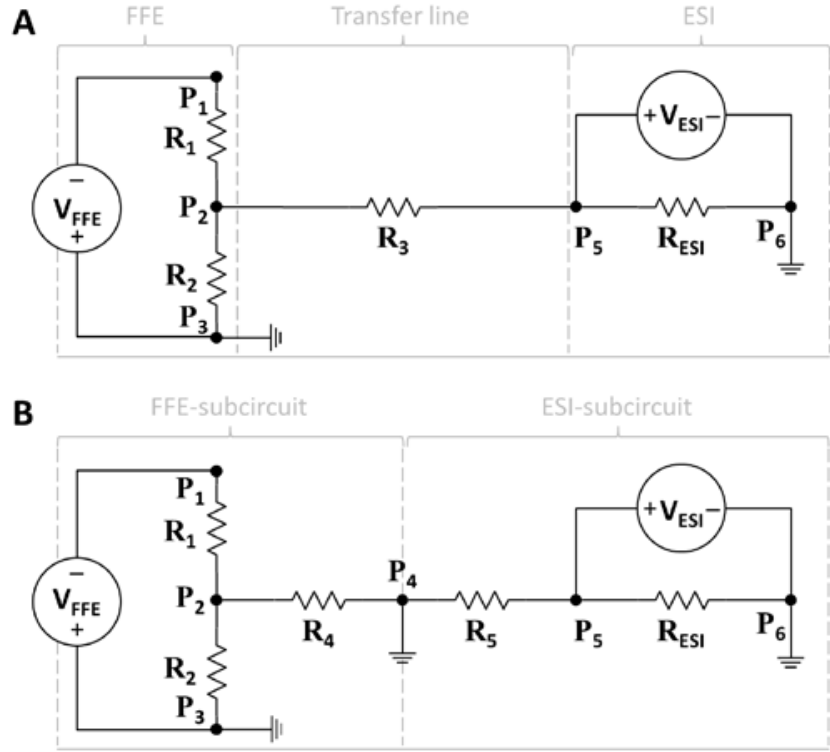

Figure S2. Electric circuit diagrams of FFE-ESI-MS depicting the situation without (A) and with (B) grounding of the transfer line.

middle between the two electrodes (i.e. $L_{1}=L_{2}$ ), $r_{\mathrm{L}}$ becomes equal to unity and $R_{1}$ and $R_{2}$ become equal to each other. For outlet 1 and 3 in our setup, $r_{\mathrm{L}}$ is $0.46\left(L_{1}=3.15 \mathrm{~cm}\right.$, $\left.L_{2}=6.85 \mathrm{~cm}\right)$ and $2.17\left(L_{1}=6.85 \mathrm{~cm}, L_{2}=3.15 \mathrm{~cm}\right)$, respectively. For all following equations, we assume that $\mathrm{P}_{2}$ is always located between $\mathrm{P}_{1}$ and $\mathrm{P}_{3}$ (i.e. $\mathrm{P}_{1} \neq \mathrm{P}_{2} \neq \mathrm{P}_{3}$ ), so that $R_{1}>0$ and $R_{2}>0$. Using $r_{\mathrm{L}}, R_{1}$ can be expressed by a fraction of the total resistance $R$ in the FFE chip:

$$
R_{1}=\frac{r_{\mathrm{L}}}{1+r_{\mathrm{L}}} R
$$

$R_{2}$ is simply $R-R_{1}$. If $\mathrm{P}_{2}$ is exactly in the middle between the two electrodes, the resistances $R_{1}$ and $R_{2}$ are exactly half of the total resistance $R(32.6 \mathrm{k} \Omega)$, namely $R_{1}=R_{2}=16.3 \mathrm{k} \Omega$. The remaining resistances $R_{3}, R_{4}$ and $R_{5}$ can be calculated using Equation (S1) whilst taking into account the tubing dimensions: diameter of $1 / 16 ”=1.5875 \mathrm{~mm}$ (resulting in a crosssection area of $1.979 \mathrm{~mm}^{2}$ ) and lengths of 38, 24, and $14 \mathrm{~cm}$ for $R_{3}, R_{4}$, and $R_{5}$, respectively.

Circuit of a sole FFE chip. No matter where $\mathrm{P}_{2}$ is located, the currents can be calculated by using Kirchhoff's circuit laws as:

$$
I_{\mathrm{R}_{1}}=I_{\mathrm{R}_{2}}=\frac{U_{21}}{R_{1}}=\frac{U_{32}}{R_{2}}=\frac{U_{31}}{R_{1}+R_{2}}=\frac{U_{31}}{R}=I_{\text {avg }}
$$

where $I_{\mathrm{Rx}}$ and $R_{\mathrm{x}}$ are the current through and the resistance of the corresponding resistor. $U_{\mathrm{xy}}$ is the potential difference between points $\mathrm{P}_{\mathrm{x}}$ and $\mathrm{P}_{\mathrm{y}}$ :

$U_{\mathrm{xy}}=P_{\mathrm{x}}-P_{\mathrm{y}}$

This, of course, should result in a current of $23.0 \mathrm{~mA}$ since this is just the back-calculation of our measurement, which was used to determine the conductivity of the electrolyte. From Equation (S4) we can find an expression for $P_{2}$ : 


$$
\begin{array}{ll}
\frac{U_{21}}{R_{1}} & =\frac{U_{32}}{R_{2}} \\
U_{21} R_{2} & =U_{32} R_{1} \\
R_{2} P_{2}-R_{2} P_{1} & =R_{1} P_{3}-R_{1} P_{2} \\
P_{2}\left(R_{2}+R_{1}\right) & =R_{1} P_{3}+R_{2} P_{1}
\end{array}
$$

This leads to the following general expression for $P_{2}$ in a sole chip:

$$
P_{2}=\frac{R_{2} P_{1}+R_{1} P_{3}}{R_{1}+R_{2}}
$$

We can express $R_{1}$ by $r_{\mathrm{L}}$ and $R_{2}$ using Equation (S2):

$$
P_{2}=\frac{R_{2} P_{1}+r_{\mathrm{L}} R_{2} P_{3}}{r_{\mathrm{L}} R_{2}+R_{2}}=\frac{P_{1}+r_{\mathrm{L}} P_{3}}{r_{\mathrm{L}}+1}
$$

In our case $P_{3}$ is grounded and its potential $P_{3}$ is zero. Therefore, $P_{2}$ becomes a simple fraction of $P_{1}$ :

$$
P_{2}^{\text {sole }}=\frac{1}{r_{\mathrm{L}}+1} P_{1}
$$

The resulting equation is very simple and easy to interpret. For example, if $\mathrm{P}_{2}$ is located in the middle between the two electrodes (i.e. $r_{\mathrm{L}}=1$ ), its potential $P_{2}$ becomes exactly half of the potential at $P_{1}$.

FFE-MS circuit without grounding (Figure S3A). All resistances and all potentials except for potential $P_{2}$ are known. There is a large, positive potential at $\mathrm{P}_{5}$, a zero potential at $\mathrm{P}_{6}$, another zero potential at $\mathrm{P}_{3}$, and a zero or negative potential at $P_{1}$. Therefore, the current flows from $P_{5}$ towards $P_{1}$ (or $P_{6}$ ) and from $\mathrm{P}_{3}$ towards $\mathrm{P}_{1}$. This results in the following relationship of the currents:

$$
I_{\mathrm{R}_{1}}=I_{\mathrm{R}_{2}}+I_{\mathrm{R}_{3}}
$$

The currents $I_{\mathrm{R}_{1}}, I_{\mathrm{R}_{2}}$, and $I_{\mathrm{R}_{3}}$ can be expressed via the potential differences and resistances:

$\frac{U_{21}}{R_{1}}=\frac{U_{32}}{R_{2}}+\frac{U_{52}}{R_{3}}$

or potential values using Equation (S5):

$$
\frac{P_{2}-P_{1}}{R_{1}}=\frac{P_{3}-P_{2}}{R_{2}}+\frac{P_{5}-P_{2}}{R_{3}}
$$

We can solve Equation (S11) for $P_{2}$ :

$$
\begin{aligned}
& \frac{R_{2} R_{3}\left(P_{2}-P_{1}\right)-R_{1} R_{3}\left(P_{3}-P_{2}\right)-R_{1} R_{2}\left(P_{5}-P_{2}\right)}{R_{1} R_{2} R_{3}}=0 \\
& R_{2} R_{3}\left(P_{2}-P_{1}\right)-R_{1} R_{3}\left(P_{3}-P_{2}\right)-R_{1} R_{2}\left(P_{5}-P_{2}\right)=0 \\
& R_{2} R_{3} P_{2}+R_{1} R_{3} P_{2}+R_{1} R_{2} P_{2}=R_{1} R_{3} P_{3}+R_{1} R_{2} P_{5}+R_{2} R_{3} P_{1} \\
& P_{2}\left(R_{2} R_{3}+R_{1} R_{3}+R_{1} R_{2}\right)=R_{1} R_{3} P_{3}+R_{1} R_{2} P_{5}+R_{2} R_{3} P_{1}
\end{aligned}
$$

Finally, we get a general expression for $P_{2}$ :

$$
P_{2}=\frac{R_{2} R_{3} P_{1}+R_{1} R_{3} P_{3}+R_{1} R_{2} P_{5}}{R_{2} R_{3}+R_{1} R_{3}+R_{1} R_{2}}
$$

We can express $R_{1}$ via $r_{\mathrm{L}}$ and $R_{2}$ using Equation (S2):

$$
\begin{aligned}
P_{2} & =\frac{R_{2} R_{3} P_{1}+r_{\mathrm{L}} R_{2} R_{3} P_{3}+r_{\mathrm{L}} R_{2}^{2} P_{5}}{R_{2} R_{3}+r_{\mathrm{L}} R_{2} R_{3}+r_{\mathrm{L}} R_{2}^{2}}= \\
& =\frac{R_{3} P_{1}+r_{\mathrm{L}} R_{3} P_{3}+r_{\mathrm{L}} R_{2} P_{5}}{R_{3}+r_{\mathrm{L}} R_{3}+r_{\mathrm{L}} R_{2}}= \\
& =\frac{R_{3} \frac{P_{1}+r_{\mathrm{L}} P_{3}}{r_{\mathrm{L}}+1}+\frac{r_{\mathrm{L}}}{r_{\mathrm{L}}+1} R_{2} P_{5}}{R_{3}+\frac{r_{\mathrm{L}}}{r_{\mathrm{L}}+1} R_{2}}
\end{aligned}
$$

The first term in the numerator resembles the term of $P_{2}$ for the sole chip in Equation (S7). Therefore, we factorize the whole fraction as follows:

$$
\begin{aligned}
P_{2}= & \frac{P_{1}+r_{\mathrm{L}} P_{3}}{r_{\mathrm{L}}+1} \frac{R_{3}+\frac{r_{\mathrm{L}}+1}{P_{1}+r_{2} P_{3}} \frac{r_{\mathrm{L}}}{r_{\mathrm{L}}+1} R_{2} P_{5}}{R_{3}+\frac{r_{\mathrm{L}}}{r_{\mathrm{L}}+1} R_{2}} \\
= & \frac{P_{1}+r_{\mathrm{L}} P_{3}}{r_{\mathrm{L}}+1} \frac{1+\frac{P_{5}}{P_{1}+r_{\mathrm{L}} P_{3}} \frac{r_{\mathrm{L}} R_{2}}{R_{3}}}{1+\frac{1}{r_{\mathrm{L}}+1} \frac{r_{\mathrm{L}} R_{2}}{R_{3}}}
\end{aligned}
$$

From this equation it is easy to see that the very first factor is indeed the expression for $P_{2}$ of the sole chip in Equation S7. Again, in our case $\mathrm{P}_{3}$ is grounded and its potential $P_{3}$ is zero. Furthermore, $r_{\mathrm{L}} R_{2}$ can be replaced by $R_{1}$ giving the following expression for $P_{2}$ in the non-grounded system:

$$
P_{2}^{\mathrm{ng}}=P_{2}^{\text {sole }} \frac{1+\frac{P_{5}}{P_{1}} \frac{R_{1}}{R_{3}}}{1+\frac{1}{r_{\mathrm{L}}+1} \frac{R_{1}}{R_{3}}}=P_{2}^{\text {sole }} T^{\mathrm{ng}}
$$

The currents can then be calculated using the corresponding potential differences and resistances.

Difference in $\boldsymbol{P}_{2}$ between setup without grounding and sole chip. Furthermore, Equation (S13) reveals the difference between the sole chip and the non-grounded, hyphenated setup. The difference (influence) is expressed by the modifierterm $T^{\text {ng }}$ multiplied to $P_{2}{ }^{\text {sole }}$. Because of this term, the potential $P_{2}{ }^{\text {ng }}$ depends on the ESI potential $P_{5}$ and the resistance $R_{3}$ of the transfer line. If we look closely at both the numerator and denominator in Equation (S13), it becomes clear that they only differ in the factor in front of the ratio of resistances. Given that the ESI potential (here: $5.5 \mathrm{kV}$ ) is practically always a lot larger than the FFE potential (here: $-750 \mathrm{~V}$ ) and $r_{\mathrm{L}}$ is a positive, real number, we get the following expressions for both ratios: 


$$
\frac{P_{5}}{P_{1}}<-1 \quad 0<\frac{1}{r_{\mathrm{L}}+1}<1
$$

Furthermore, the resistances $R_{1}$ and $R_{3}$ are always positive, real numbers. $R_{3}$ can be considered much larger than $R_{1}$ given that the resistance of the transfer line is larger than of the FFE chip.

$0 \leq \frac{R_{1}}{R_{3}} \ll 1$

This assumption is reasonable since the cross-section of the tubing is a lot less than the cross-section of the FFE chip. Due to all this, $T^{\text {ng }}$ gives a value between zero and one, i.e. the connected ESI source reduces the absolute value of $P_{2}$ compared to the sole chip. In the ideal case, it would be approaching unity meaning that the ESI source has no influence on FFE. For this, the denominator and the numerator have to be equal:

$$
\begin{aligned}
& 1+\frac{P_{5} R_{1}}{P_{1} R_{3}}=1+\frac{1}{r_{\mathrm{L}}+1} \frac{R_{1}}{R_{3}} \\
& \frac{P_{5}}{P_{1}}=\frac{1}{r_{\mathrm{L}}+1} \\
& P_{5}\left(r_{\mathrm{L}}+1\right)=P_{1}
\end{aligned}
$$

Again, $r_{\mathrm{L}}$ is a positive, real number, i.e. the expression $r_{\mathrm{L}}+1$ is always greater or equal to 1 . This means that i) $P_{1}$ and $P_{5}$ have to have the same sign, ii) $P_{1}$ (FFE potential) has actually to be a multiple of $P_{5}$ (ESI potential) for $T^{\text {ng }}$ to approach unity. Furthermore, the ratio of both potentials depends on the outlet connected to the ESI interface. Thus, it is practically impossible to fulfill the criteria given in Equation (S14).

FFE-MS circuit with grounding (Figure S3B). Introduced grounding at $\mathrm{P}_{4}$ divides the circuit into two subcircuits, which can be calculated independently. Again, all resistances and all potentials except for potential $P_{2}$ are known. The ESI subcircuit is very easy to calculate. The current of $I_{\mathrm{R}_{5}}$ can be easily expressed by the following relation. Since $\mathrm{P}_{4}$ is grounded its potential $P_{4}$ is zero:

$$
I_{\mathrm{R}_{5}}=\frac{U_{54}}{R_{5}}=\frac{P_{5}-P_{4}}{R_{5}}=\frac{P_{5}}{R_{5}}
$$

The currents in the FFE subcircuit are as follows:

$$
I_{\mathrm{R}_{1}}=I_{\mathrm{R}_{2}}+I_{\mathrm{R}_{4}}
$$

The currents $I_{\mathrm{R}_{1}}, I_{\mathrm{R}_{2}}$, and $I_{\mathrm{R}_{4}}$ can be expressed by the potential differences and resistances

$\frac{U_{21}}{R_{1}}=\frac{U_{32}}{R_{2}}+\frac{U_{42}}{R_{4}}$

and the potential differences can be expressed by the actual potentials using Equation (S5):

$$
\frac{P_{2}-P_{1}}{R_{1}}=\frac{P_{3}-P_{2}}{R_{2}}+\frac{P_{4}-P_{2}}{R_{4}}
$$

We can solve Equation (S18) for $P_{2}$ :

$$
\begin{aligned}
& \frac{R_{2} R_{4}\left(P_{2}-P_{1}\right)-R_{1} R_{4}\left(P_{3}-P_{2}\right)-R_{1} R_{2}\left(P_{4}-P_{2}\right)}{R_{1} R_{2} R_{4}}=0 \\
& R_{2} R_{4}\left(P_{2}-P_{1}\right)-R_{1} R_{4}\left(P_{3}-P_{2}\right)-R_{1} R_{2}\left(P_{4}-P_{2}\right)=0 \\
& R_{2} R_{4} P_{2}+R_{1} R_{4} P_{2}+R_{1} R_{2} P_{2}=R_{1} R_{4} P_{3}+R_{1} R_{2} P_{4}+R_{2} R_{4} P_{1} \\
& P_{2}\left(R_{2} R_{4}+R_{1} R_{4}+R_{1} R_{2}\right)=R_{1} R_{4} P_{3}+R_{1} R_{2} P_{4}+R_{2} R_{4} P_{1}
\end{aligned}
$$

Finally, we get a general expression for $P_{2}$ :

$$
P_{2}=\frac{R_{2} R_{4} P_{1}+R_{1} R_{4} P_{3}+R_{1} R_{2} P_{4}}{R_{2} R_{4}+R_{1} R_{4}+R_{1} R_{2}}
$$

We can express $R_{1}$ by $r_{\mathrm{L}}$ and $R_{2}$ using Equation (S2):

$$
\begin{aligned}
P_{2} & =\frac{R_{2} R_{4} P_{1}+r_{L} R_{2} R_{4} P_{3}+r_{L} R_{2}^{2} P_{4}}{R_{2} R_{4}+r_{L} R_{2} R_{4}+r_{L} R_{2}^{2}}= \\
& =\frac{R_{4} P_{1}+r_{L} R_{4} P_{3}+r_{L} R_{2} P_{4}}{R_{4}+r_{L} R_{4}+r_{L} R_{2}}= \\
& =\frac{R_{4} \frac{P_{1}+r_{L} P_{3}}{r_{L}+1}+\frac{r_{L}}{r_{L}+1} R_{4} P_{4}}{R_{4}+\frac{r_{L}}{r_{L}+1} R_{2}}
\end{aligned}
$$

The first term in the numerator resembles the term of $P_{2}$ for the sole chip in Equation (S7). Therefore, we factorize the whole fraction as follows:

$$
\begin{aligned}
P_{2} & =\frac{P_{1}+r_{L} P_{3}}{r_{L}+1} \frac{R_{4}+\frac{r_{L}+1}{P_{1}+r_{2} P_{3}} \frac{r_{L}}{r_{L}+1} R_{2} P_{4}}{R_{4}+\frac{r_{L}}{r_{L}+1} R_{2}}= \\
& =\frac{P_{1}+r_{L} P_{3}}{r_{L}+1} \frac{1+\frac{P_{4}}{P_{1}+r_{L} P_{3}} \frac{r_{L} R_{2}}{R_{4}}}{1+\frac{1}{r_{L}+1} \frac{r_{L} R_{2}}{R_{4}}}
\end{aligned}
$$

From this equation it is easy to see that the very first factor is indeed the expression for $P_{2}$ of the sole chip in Equation S7. Furthermore, this equation and its derivation equal the ones for the non-grounded system (see last subsection). As before, $\mathrm{P}_{3}$ is grounded and its potential $P_{3}$ is zero. Furthermore, $r_{\mathrm{L}} R_{2}$ can be replaced by $R_{1}$. The key difference is that $\mathrm{P}_{4}$ is grounded, i.e. its potential is zero. This leads to the following general expression for $P_{2}$ in the applied-grounded system:

$$
P_{2}^{\text {ag }}=P_{2}^{\text {sole }} \frac{1}{1+\frac{1}{r_{L}+1} \frac{R_{1}}{R_{4}}}=P_{2}^{\text {sole }} T^{\text {ag }}
$$

The currents can then be calculated using the corresponding potential differences and resistances.

Difference in $\boldsymbol{P}_{2}$ between all three setups. Similar to the nongrounded case, Equation (S20) reveals the difference between 
the sole chip and the applied-grounded, hyphenated setup. The difference is expressed by the modifier-term $T^{\mathrm{ag}}$ multiplied to $P_{2}{ }^{\text {sole }}$. However, in contrast to $T^{\text {ng }}$, which is expressed in Equation (S13), $T^{\mathrm{ag}}$ has 'lost' its numerator and, thus, does not depend on the ESI potential anymore. Still, there is a dependency on the resistance $R_{4}$ of the transfer line. However, we can make the assumption that the resistance of the transfer line is much larger than of the FFE chip. Therefore, the denominator of $T^{\mathrm{ag}}$ and, in turn, $T^{\mathrm{ag}}$ itself approaches unity:
Conclusively, applied grounding renders the influence of the hyphenation on the potential $P_{2}$ negligible:

$$
P_{2}^{\text {ag }} \approx P_{2, \text { approx }}^{\text {ag }}=P_{2}^{\text {sole }} T_{\text {approx }}^{\text {ag }}=P_{2}^{\text {sole }}
$$

Calculated values of circuits. All values calculated with the above formulas above are listed in Table S2. For discussion, see main text.

Table S1. Calculated values of electrical potentials and currents for electric circuits in Figure S3 with $\mathrm{P}_{2}$ being the sample outlet 2 . The potentials at $\mathrm{P}_{3}, \mathrm{P}_{6}$, and $\mathrm{P}_{4}$ (when present) are always $0 \mathrm{~V}$ and, therefore, not listed. ${ }^{\mathrm{a}}$ Calculated values for an FFE chip without any additional connections or hyphenations for comparison. ${ }^{b}$ For clarity, the absolute values of the currents are given only.

\begin{tabular}{|c|c|c|c|c|c|c|c|c|c|c|c|}
\hline \multirow{2}{*}{\multicolumn{2}{|c|}{ Configuration }} & \multicolumn{5}{|c|}{ Voltages and potentials, V } & \multicolumn{5}{|c|}{ Current $^{\mathrm{b}}, \mathrm{mA}$} \\
\hline & & $V_{\mathrm{FFE}}$ & $V_{\mathrm{ESI}}$ & $P_{1}$ & $P_{2}$ & $P_{5}$ & $I_{\mathrm{R}_{1}=16.3 \mathrm{k} \Omega}$ & $I_{\mathrm{R}_{2}}=16.3 \mathrm{k} \Omega$ & $I_{\mathrm{R}_{3}=1.13 \mathrm{M} \Omega}$ & $I_{\mathrm{R}_{4}=713 \mathrm{k} \Omega}$ & $I_{\mathrm{R}_{5}=416 \mathrm{k} \Omega}$ \\
\hline 1 & sole FFE chip ${ }^{a}$ & 750 & - & -750 & -375 & - & 23.0 & 23.0 & - & - & - \\
\hline 2 & Fig. 3A (no ground) & - & 5500 & 0 & 39 & 5500 & 2.4 & 2.4 & 4.7 & - & - \\
\hline 3 & Fig. 3A (no ground) & 750 & 5500 & -750 & -334 & 5500 & 25.5 & 20.4 & 5.0 & - & - \\
\hline 4 & Fig. 3B (grounding) & - & 5500 & 0 & 0 & 5500 & 0 & 0 & - & 0 & 13.2 \\
\hline 5 & Fig. 3B (grounding) & 750 & 5500 & -750 & -371 & 5500 & 23.2 & 22.7 & - & 0.5 & 13.2 \\
\hline
\end{tabular}

$T^{\mathrm{ag}} \approx T_{\mathrm{approx}}^{\mathrm{ag}}=1(\mathrm{~S} 21)$ 


\section{CALCULATING DESCRIPTORS $H_{\text {ASYM }}$ AND $H_{\text {LEAK }}$}

In order to find dependencies of and solutions to eliminate the described interferences (see main text), we examine them mathematically. For this, we introduce two descriptors.

Descriptor of potential asymmetry. First, we define $H_{\text {asym }}$ as a measure of the asymmetry of potential distribution in the FFE chip as

$$
H_{\text {asym }}=\frac{U_{21}}{U_{32}}-r_{L}
$$

The ratio of potential differences can be expressed by the potentials themselves using Equation (S5):

$\frac{U_{21}}{U_{32}}=\frac{P_{2}-P_{1}}{P_{3}-P_{2}}$

The potential at $\mathrm{P}_{3}$ is zero since it is grounded. Substituting this expression into Equation (S23) gives:

$$
H_{\text {asym }}=\frac{P_{2}-P_{1}}{-P_{2}}-r_{L}=\frac{P_{1}}{P_{2}}-\left(r_{L}+1\right)
$$

$H_{\text {asym }}$ is zero in a non-disturbed FFE-system (i.e. no asymmetry), which indicates the absence of any shifting effects as it is the case of the sole FFE chip (see below). For disturbed systems, the asymmetry factor deviates from zero and becomes a measure for the disturbance of the system.

Potential asymmetry in the sole chip. For the sole chip we can replace the second term of Equation (S25) by using Equation (S8) giving

$$
H_{\text {asym }}^{\text {sole }}=\frac{P_{1}}{P_{2}^{\text {sole }}}-\frac{P_{1}}{P_{2}^{\text {sole }}}=0
$$

Obviously, there is no hyphenation-induced potential asymmetry in the sole chip because there is no hyphenation, i.e. $H_{\text {asym }}^{\text {sole }}$ is always zero.

Potential asymmetry in non-grounded setup. The potential $P_{2}$ in Equation (S25) can be expressed by $P_{2}{ }^{n g}$ from Equation (S13):

$$
H_{\mathrm{asym}}^{\mathrm{ng}}=\frac{P_{1}}{P_{2}^{\mathrm{ng}}}-\left(r_{L}+1\right)=\frac{P_{1}}{P_{2}^{\text {sole }}} \frac{1}{T^{\mathrm{ng}}}-\left(r_{L}+1\right)
$$

Plugging in the expression for $P_{1}$ over $P_{2}^{\text {sole }}$ from Equation (S8) gives

$$
H_{\mathrm{asym}}^{\mathrm{ng}}=\left(r_{L}+1\right) \frac{1}{T^{\mathrm{ng}}}-\left(r_{L}+1\right)
$$

Plugging in the expression for $T^{\mathrm{ng}}$ from Equation (S13) gives after some rearranging a general expression for the asymmetric descriptor in the non-grounded setup:

$$
\begin{aligned}
H_{\mathrm{asym}}^{\mathrm{ng}}= & \frac{\left(r_{L}+1\right)\left(1+\frac{1}{r_{L}+1} \frac{R_{1}}{R_{3}}\right)}{1+\frac{P_{5}}{P_{1}} \frac{R_{1}}{R_{3}}}-\left(r_{L}+1\right) \\
= & \frac{r_{L}+1+\frac{R_{1}}{R_{3}}}{1+\frac{P_{5}}{P_{1}} \frac{R_{1}}{R_{3}}}-\left(r_{L}+1\right) \\
= & \frac{r_{L}+1+\frac{R_{1}}{R_{3}}-\left(r_{L}+1\right)-\left(r_{L}+1\right) \frac{P_{5}}{P_{1}} \frac{R_{1}}{R_{3}}}{R_{3}}
\end{aligned}=
$$

$H_{\text {asym }}{ }^{\text {ng }}$ now depends solely on the ratio of $R_{3}$ over $R_{1} \cdot r_{\mathrm{L}}$ is a parameter describing the position of $\mathrm{P}_{2}$. In theory, $H_{\text {asym }}$ ng depends also on the ratio of the two potentials at $\mathrm{P}_{1}$ and $\mathrm{P}_{5}$. However, influencing the descriptor by adjusting the potentials is very limited since the potentials have to be in certain ranges for the FFE-MS tandem to be useful. Thus, this ratio is considered to be a constant. Further, the resistances can be expressed by

$R_{\mathrm{n}}=\frac{1}{\kappa} \frac{L_{\mathrm{n}}}{A_{\mathrm{n}}} \equiv \frac{G_{\mathrm{n}}}{\kappa}$

where $A_{\mathrm{n}}$ and $L_{\mathrm{n}}$ are the area of the cross-section and the length of the conductor, respectively, $\kappa$ is the conductivity of the BGE, and $G_{\mathrm{n}}$ is the geometry factor (the ratio of $L_{\mathrm{n}}$ over $A_{\mathrm{n}}$ ). Conductivity $\kappa$ is the same for both resistors since it is the same BGE flowing through the FFE as well as the tubing. Thus, it cancels itself out when substituting the resistances by Equation (S26). This means that $H_{\text {asym }}{ }^{\text {ng }}$ only depends on the ratio of the geometry factors $G_{1}$ and $G_{3}$.

$$
H_{\mathrm{asym}}^{\mathrm{ng}}=\frac{1-\left(r_{L}+1\right) \frac{P_{5}}{P_{1}}}{\frac{G_{3}}{G_{1}}+\frac{P_{5}}{P_{1}}}
$$

Since $G_{1}$ describes the geometry of the FFE chip and $G_{3}$ the geometry of the transfer line (from $\mathrm{P}_{2}$ to $\mathrm{P}_{5}$ ), we replace them 
by the more descriptive symbols of $G_{\mathrm{FFE}}$ and $G_{\mathrm{trans}}$, respectively:

$$
H_{\text {asym }}^{\mathrm{ng}}=\frac{1-\left(r_{L}+1\right) \frac{P_{5}}{P_{1}}}{\frac{G_{\text {trans }}}{G_{\mathrm{FFE}}}+\frac{P_{5}}{P_{1}}}
$$

It is important to point out that due to this, asymmetric potential distribution would also happen when a low conductive electrolyte, such as acetic acid, is used for a production-scale setup. $H_{\text {asym }}^{\text {ng }}$ can be seen as a function of the ratio of geometry factors $G_{\mathrm{FFE}}$ and $G_{\text {trans }}$ :

$$
H_{\mathrm{asym}}^{\mathrm{ng}}(x) \equiv \frac{1-\left(r_{L}+1\right) \frac{P_{5}}{P_{1}}}{\frac{1}{x}+\frac{P_{5}}{P_{1}}}
$$

where $x$ is:

$$
\frac{G_{\mathrm{FFE}}}{G_{\text {trans }}} \equiv X
$$

The shape of the function depends on the position of the outlet, which is described by the parameter $r_{\mathrm{L}}$ (Figure S4).

Potential asymmetry in grounded setup. The potential $P_{2}$ in Equation (S25) can be expressed by $P_{2}{ }^{\text {ag }}$ from Equation (S20):

$$
H_{\text {asym }}^{\text {ag }}=\frac{P_{1}}{P_{2}}-\left(r_{L}+1\right)=\frac{P_{1}}{P_{2}^{\text {sole }} T^{\text {ag }}}-\left(r_{L}+1\right)
$$

Plugging in the expression for $P_{1}$ over $P_{2}^{\text {sole }}$ from Equation (S8) gives

$$
H_{\mathrm{asym}}^{\mathrm{ag}}=\left(r_{L}+1\right) \frac{1}{T^{\mathrm{ag}}}-\left(r_{L}+1\right)
$$

Plugging in the expression for $T^{\mathrm{ag}}$ from Equation (S20) gives after some rearranging a general expression for the asymmetric descriptor in the applied-grounded setup:

$$
\begin{aligned}
H_{\mathrm{asym}}^{\mathrm{ag}} & =\left(r_{L}+1\right)\left(1+\frac{1}{r_{L}+1} \frac{R_{1}}{R_{4}}\right)-\left(r_{L}+1\right)= \\
& =r_{L}+1+\frac{R_{1}}{R_{4}}-\left(r_{L}+1\right)=\frac{R_{1}}{R_{4}}
\end{aligned}
$$

Again, if we substitute the resistances $R_{1}$ and $R_{4}$ by Equation (S26) we see that the descriptor only depends on the ratio of the geometry factors:

$$
H_{\mathrm{asym}}^{\mathrm{ag}}=\frac{G_{1}}{G_{4}}
$$

Since $G_{1}$ describes the geometry of the FFE chip and $G_{4}$ the geometry of the transfer line (from $\mathrm{P}_{2}$ to $\mathrm{P}_{4}$ ), we replace them - analogously to $H_{\text {asym }}{ }^{\text {ng }}$ - by the more descriptive symbols of $G_{\mathrm{FFE}}$ and $G_{\text {trans }}$, respectively:
$H_{\mathrm{asym}}^{\mathrm{ag}}=\frac{G_{\mathrm{FFE}}}{G_{\text {trans }}}$

Similar to $H_{\text {asym }}{ }^{\text {ng }}$, the conductivity of the BGE plays no role for the asymmetric potential distribution. In contrast, however, $H_{\text {asym }}{ }^{\text {ag }}$ has no - even theoretical - dependency on the potentials and the dependency on the ratio of geometry factors is very simple - linear proportional. Again, $H_{\text {asym }}^{\text {ag }}$ can be seen as function of the ratio of geometry factors $G_{\mathrm{FFE}}$ and $G_{\text {trans }}$ :

$$
H_{\mathrm{asym}}^{\mathrm{ag}}(x) \equiv x
$$

where $x$ is the same as for $H_{\text {asym }}{ }^{\text {ng. }}$

$$
\frac{G_{\mathrm{FFE}}}{G_{\text {trans }}} \equiv \chi
$$

$H_{\text {asym }}{ }^{\text {ag }}$ has no parameter and, therefore, its shape does not depend on the position of the outlet.

Comparing $\boldsymbol{H}_{\text {asym }}$ for non-grounded and grounded setup. The descriptor for the asymmetrical potential distribution is quite different for both setups. However, in both cases the main contributor is the ratio of geometry factors of the FFE chip and the transfer line $\left(G_{\mathrm{FFE}} / G_{\text {trans }}=x\right)$. Therefore, we describe both descriptors as functions of $x$ in order to compare and examine the influence of grounding. Applying grounding to the setup means to exchange $H_{\text {asym }}{ }^{\text {ng }}$ by $H_{\text {asym }}{ }^{\text {ag }}$, i.e. the type of function changes dramatically with the state of grounding. It is a steeply growing function $H_{a s y m}{ }^{\text {ng }}$ when no grounding is applied (see Equation (S28) and Figure S4); small changes in $x$ can lead to very large changes in symmetry. In contrast to $H_{\text {asym }}{ }^{\text {ng }}, H_{\text {asym }}{ }^{\text {ag }}$ is a linear function (see Equation (S30)); it is less sensitive to changes in $x$. Both functions are plotted in Figure S4.

It is clear that the primary strategy to minimize $H_{\text {asym }}$ is to increase the geometry factor of the transfer line $\left(G_{\text {trans }}\right)$ as much as possible relative to the geometry factor of FFE ( $\left.G_{\mathrm{FFE}}\right)$. When grounding is applied, $G_{\text {trans }}$ decreases and $G_{\mathrm{FFE}}$ does not change; however, $H_{\text {asym }}$ still decreases significantly (from 0.25 to 0.023 in our example, see inset of Figure S4) due to the change of function $H_{\text {asym }}(x)$. In our specific case, $H_{\text {asym }}^{\text {ag }}=0.023$ suggests that the potential distribution in the chip is almost perfectly symmetrical, and further modifications to the setup are not necessary. Therefore, grounding is the most straightforward and most practical way of ensuring a symmetrical potential distribution in FFE.

Descriptor for current leak at the ESI interface. We define a second descriptor, $H_{\text {leak }}$, as a measure of current leak from the ESI probe $\left(\mathrm{P}_{5}\right)$ into the transfer line:

$$
H_{\text {leak }}=\frac{I_{\mathrm{R}_{\text {trans }}}}{I_{\mathrm{R}_{\mathrm{ESI}}}}=\frac{R_{\mathrm{ESI}} U_{\text {trans }}}{R_{\text {trans }} U_{\text {ESI }}}(\mathrm{S} 31)
$$

$H_{\text {leak }}$ is zero if there is no current leak and non-zero otherwise.

Current leak in non-grounded setup. For this setup, $H_{\text {leak }}$ corresponds to the geometry factor $G_{3}$ as well as the $P_{2}{ }^{\text {ng }}, P_{5}$, and $P_{6}$ : 


$$
H_{\text {leak }}^{\text {ng }}=\frac{I_{\mathrm{R}_{3}}^{\mathrm{ng}}}{I_{\mathrm{R}_{\mathrm{ESI}}}}=\frac{R_{\mathrm{ESI}} U_{52}}{R_{3} U_{56}}=R_{\mathrm{ESI}} \frac{\kappa}{G_{3}} \frac{P_{5}-P_{2}^{n g}}{P_{5}-P_{6}}
$$

Since $\mathrm{P}_{6}$ is grounded its potential is zero.

Current leak in the applied-grounded setup. Here, $H_{\text {leak }}$ corresponds to the geometry factor of $G_{5}$ :

$$
H_{\text {leak }}^{\mathrm{ag}}=\frac{I_{\mathrm{R}_{5}}^{\mathrm{ag}}}{I_{\mathrm{R}_{\mathrm{ESI}}}}=\frac{R_{\mathrm{ESI}} U_{54}}{R_{5} U_{56}}=R_{\mathrm{ESI}} \frac{\kappa}{G_{5}} \frac{P_{5}-P_{4}}{P_{5}-P_{6}}
$$

Since $\mathrm{P}_{4}$ and $\mathrm{P}_{6}$ are grounded their potentials are zero.

Comparing $H_{\text {leak }}$ for non-grounded and grounded setup. In order to compare $H_{\text {leak }}^{\text {ng }}$ and $H_{\text {leak }}^{\text {ag }}{ }^{\text {we de define a ratio } r_{\mathrm{f}} \text { as }}$

$r_{f}=\frac{H_{\text {leak }}^{\text {ng }}}{H_{\text {leak }}^{\text {ag }}}=\frac{G_{5}}{G_{3}} \frac{P_{5}-P_{2}^{n g}}{P_{5}}=\frac{A_{5} L_{3}}{A_{3} L_{5}} \frac{P_{5}-P_{2}^{n g}}{P_{5}}$

Grounding the transfer line does not change the type of tubing used for the transfer line. Hence, the cross-section is the same for both, i.e. $A_{3}=A_{5}$ :

$$
r_{f}=\frac{L_{3}}{L_{5}} \frac{P_{5}-P_{2}^{n g}}{P_{5}}=\frac{I_{\mathrm{R}_{3}}^{\mathrm{ng}}}{I_{\mathrm{R}_{5}}^{\mathrm{ag}}}
$$

However, its effective length is reduced greatly (by a factor of 2.7 , from 38 to $14 \mathrm{~cm}$, in our case) while the potential difference changes to a much smaller extent (5834 to $5500 \mathrm{~V}$ in our case). Thus, it is clear why the current leak increases upon applying grounding by the same factor as the decrease of the transfer line length (a factor of 2.6, from 5.0 to $13.2 \mathrm{~mA}$, in our case).

Similar to $H_{\text {asym }}, H_{\text {leak }}$ depends in both cases on the corresponding geometry factor $\left(G_{3}\right.$ or $\left.G_{5}\right)$ of the transfer line. In contrast to $H_{\text {asym }}$, however, $H_{\text {leak }}$ depends further on $\kappa$.

Significance of $\boldsymbol{H}_{\text {leak }}$. The high voltage source of an ESI interface is usually loaded with a high-impedance resistor (several $\mathrm{M} \Omega$ ) and supports currents in the nanoscale or low microscale.

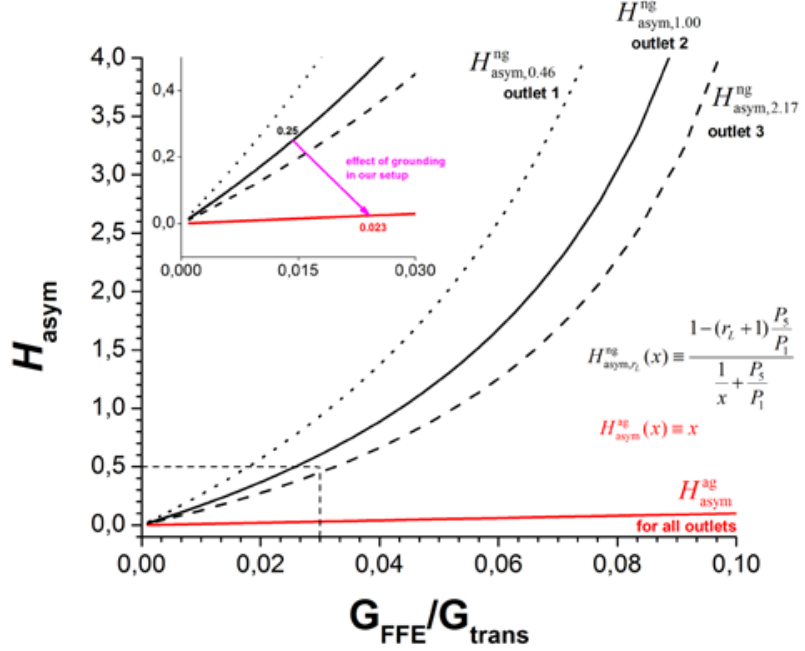

Figure S4. The two $H_{\text {asym }}$ functions, $H_{\text {asym }}^{\text {ng }}$ (shown for the corresponding $r_{\mathrm{L}}$ for outlet 1-3 of our setup) and $H_{\text {asym }}{ }^{\text {ag }}$, for the non-grounded and grounded setups, respectively. $H_{\text {asym }}{ }^{\text {ag }}$ is much flatter and insensitive to changes in the geometry factors than $\boldsymbol{H}_{\text {asym }}{ }^{\mathrm{ng}}$. The inset is an enlargement of the area marked by the dashed line. The arrow in the inset shows the change in our setup when grounding is applied for the middle outlet 2 . The shift on the $x$-axis reflects the shortening of the transfer line from 38 to $24 \mathrm{~cm}$ (to the ground).

Therefore, it most likely cannot stably maintain the potential at $\mathrm{P}_{5}$, which is one of the main factors contributing to the stability and the performance of the ESI spray itself. The higher the load - indicated by the current in the transfer line - attached to the ESI interface, the less stable is the potential at $P_{5}$.

Conclusively, while grounding improves the situation for FFE by symmetrizing the potential distribution, it impairs the situation for the ESI spray. This means that grounding is not a sufficient solution. Both descriptors, $H_{\text {asym }}$ and $H_{\text {leak }}$, have to be minimized in order to have a functional production-scale FFEMS setup. 


\section{EFFECT OF GROUNDING ON ANALYTE SIGNALS WITH NEW ESI-PROBE CONFIGURATION}

The new configuration caused the mixing of effluent and nebulizer gas before the tip of the probe. This created a microspray before the actual electrospray. A spray can be thought of as a conglomeration of droplets. Such droplets, which are surrounded by inert gas, possess a limited ability to exchange charges. Of course, they still carry charges. However, these charges can only move into direction of the physical flow of the droplets, i.e. only to the tip of the ESI probe. Thus, there is no current coming from the probe tip towards the FFE (or the ground at $\mathrm{P}_{4}$ ) anymore. This is indicated by the independence of the dye signals on the state of grounding (Figure S5).

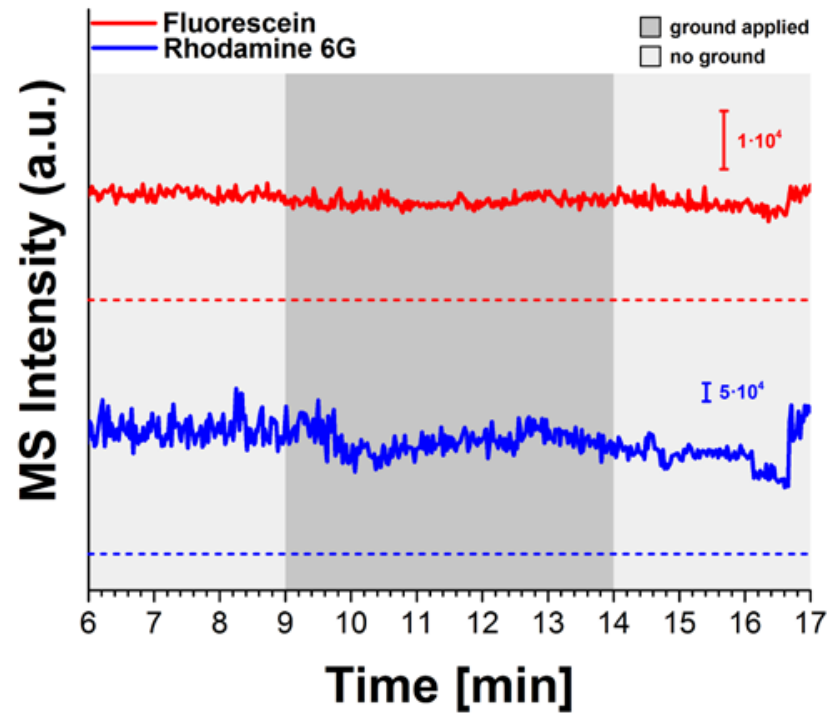

Figure S5. With this new probe configuration, the grounding state no longer influences the analyte signals; no separation voltage was applied for this experiment $\left(V_{\mathrm{FFE}}=0\right)$. The dashed lines represent the corresponding zero lines (baselines).

\section{EXAMINING THE INFLUENCE OF FFE ON ESI-SPRAY WITH NEW ESI-PROBE CONFIGURATION}

On our final setup (with the low conductive probe and withdrawn inner capillary, see main text), we conducted experiments in which we turned on $\left(V_{\mathrm{FFE}}=750 \mathrm{~V}\right)$ and off

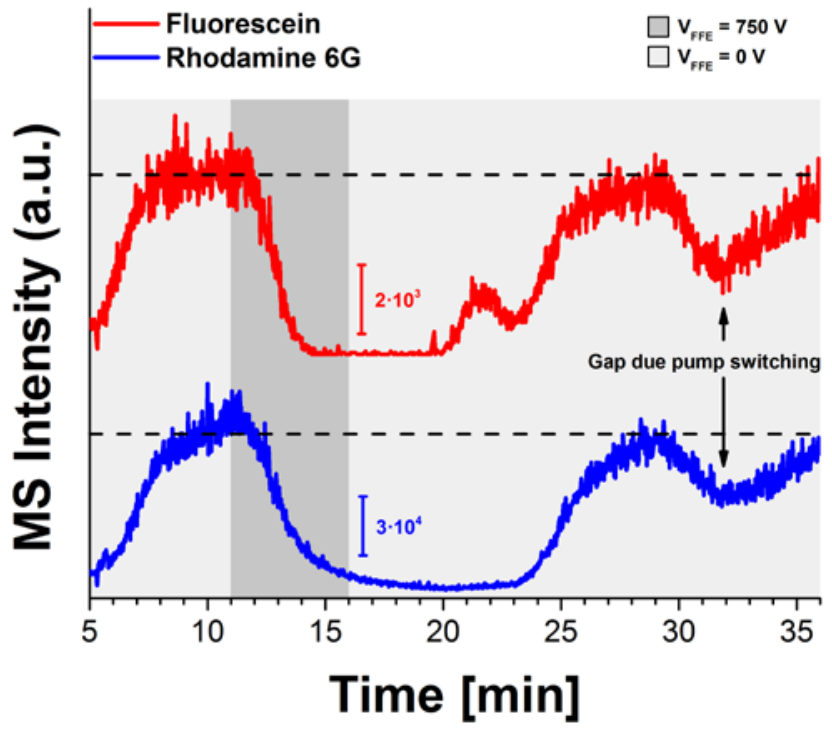

Figure S6. Influence of the separation voltage $\left(V_{F F E}\right)$ on the ESI spray. The dashed lines show the corresponding average signal at equilibrium before applying any voltage.

$\left(V_{\mathrm{FEE}}=0 \mathrm{~V}\right)$ the separation voltage in order to see if FFE has any influence on the ESI spray. An exemplary experiment is depicted in Figure S6. The experiment starts without any separation voltage, i.e. both analytes flow into outlet 2 , which is connected to the ESI interface. The signals were allowed to reach steady state (10-15 min) before turning on the separation voltage. It takes about 3-4 min for the signal to start decreasing. After about 5-7 min the signals for both analytes drop to their minima. At this point the two analytes are fully separated on the FFE chip and flow to outlets 1 and 3, respectively. After turning off the separation voltage, the analytes will again head to outlet 2 increasing the signal of both dyes again. After $13-15 \mathrm{~min}$ the signal is usually fully recovered. Furthermore, the signal to noise ratio compared to its original state is exactly the same. This indicates that neither the separation voltage itself nor turning it on or off affects the ESI spray in a quantifiable way. 


\section{PREVIOUS $\mu$ FFE-MS WORKS}

For all studies mentioned in the main text ${ }^{2-4}$, we used the same electrical circuit depicted in Figure S3A (with $\mathrm{P}_{2}$ as the middle outlet, i.e. $\left.r_{\mathrm{L}}=1.00\right)$ to calculate the currents, potentials, and descriptors. It should be noted that, while this model circuit suits our setup very well, it might not be the optimal model for the other systems. The design of the ESI sources may differ between different MS models; in particular, the ESI potential or the ground might be applied at the sprayer. Here, we calculate a worst-case scenario in which the potential is fully applied at the sprayer (capillary), i.e. it can fully 'reach' for the $\mu \mathrm{FFE}$. This model appears to be the most suitable for comparing the previous studies on $\mu \mathrm{FFE}-\mathrm{MS}$ with our study.

All used parameters extracted from the publications as well as our own (for comparison) are listed in Table S3. The geometry factors for FFE were calculated as

$$
G_{\mathrm{FFE}}=\frac{L_{\mathrm{FFE}} / 2}{A_{\mathrm{FFE}}}=\frac{L_{\mathrm{FFE}}}{2 W_{\mathrm{FFE}} h_{\mathrm{FFE}}}
$$

where $L_{\mathrm{FFE}} / 2$ is half the distance between the electrodes (since we calculate for $\mathrm{P}_{2}$ in the middle), $W_{\mathrm{FFE}}$ is the length of the electrodes, and $h_{\mathrm{FFE}}$ the height of the separation chamber. By using the reported voltages $U_{\mathrm{FFE}}$ applied and currents $I_{\mathrm{FFE}}$ observed, the conductivity of the background electrolyte (BGE) can be calculated by the following formula (see also Equation (S1)). Since the geometry factor only reflects half of the chip (see above) we have to introduce a factor of 2 here again.

$$
\kappa_{\mathrm{BGE}}=\frac{2 G_{\mathrm{FFE}}}{R_{\mathrm{FFE}}}=\frac{2 G_{\mathrm{FFE}} I_{\mathrm{FFE}}}{U_{\mathrm{FFE}}}
$$

The geometry factor for the transfer lines were calculated using

$$
G_{\text {trans }}=\frac{L_{\text {trans }}}{A_{\text {trans }}}=\frac{L_{\text {trans }}}{\pi\left(d_{\text {trans }} / 2\right)^{2}}
$$

where $L_{\text {trans }}$ and $d_{\text {trans }}$ are the length and the inner diameter of the transfer line, respectively. Benz et al. used a self-built onchip-nanosprayer. The on-chip transfer line between the separation zone and the sprayer had a width of $50 \mu \mathrm{m}$ and height of $20 \mu \mathrm{m}$, so that in this case the cross-section $A_{\text {trans }}$ is just the product of these two.

With these geometry factors and the conductivities the descriptors were calculated by using Equation S27 and S32. $110 \mathrm{M} \Omega$ was used for $R_{\mathrm{ESI}}$ to calculate $H_{\text {leak }}$.

For calculating the last two rows of Table $\mathbf{1}$ in the main tex of the manuscript, i.e. applying grounding and the modifying the ESI probe, we used Equation S29 and Equation S33 with the geometry factors for the corresponding part of the transfer line ( 0.12 for the FFE subcircuit and 0.071 for the ESI subcircuit). These factors were calculated using Equation S38 and the corresponding length of the transfer line from the FFE outlet or the ESI probe to $\mathrm{P}_{4}$, respectively $(24 \mathrm{~cm}$ for the FFE subcircuit and $14 \mathrm{~cm}$ for the ESI subcircuit).

Note: All cited studies did not report any interference between $\mu \mathrm{FFE}$ and MS. This does not mean that there were not any, which the respective authors had to overcome first to attain a working setup with the geometry and design as reported. 
Table S3. Parameters used for calculations. ${ }^{\mathrm{a}}$ Benz et al. used a self-built on-chip-nanosprayer. The on-chip transfer line between the separation zone and the sprayer had a width of $50 \mu \mathrm{m}$ and height of $20 \mu \mathrm{m}$. The cross-section $A_{\text {trans }}$ is the product of these. ${ }^{\mathrm{b}}$ This value was taken from Reference 1 not from the $\mu$ FFE-MS paper directly.

\begin{tabular}{|c|c|c|c|c|}
\hline & This work & $\begin{array}{l}\text { Ref. } 3 \\
\text { Chartogne et al. }\end{array}$ & $\begin{array}{l}\text { Ref. } 2 \\
\text { Benz et al. }\end{array}$ & $\begin{array}{l}\text { Ref. } 4 \\
\text { Park et al. }\end{array}$ \\
\hline Voltage $U_{\mathrm{FFE}}$ & $-750 \mathrm{~V}$ & $-56 \mathrm{~V}$ & $-602 \mathrm{~V}$ & $-1200 \mathrm{~V}$ \\
\hline Current $I_{\mathrm{FFE}}$ & $-23 \mathrm{~mA}$ & $-50 \mu \mathrm{A}$ & $-33 \mu \mathrm{A}$ & $-100 \mu \mathrm{A}$ \\
\hline Resistance $R_{\mathrm{FFE}}$ & $33 \mathrm{k} \Omega$ & $1.1 \mathrm{M} \Omega$ & $18 \mathrm{M} \Omega$ & $12 \mathrm{M} \Omega$ \\
\hline $\begin{array}{l}\text { FFE Dimensions } \\
\left(W_{\mathrm{FFE}} \times L_{\mathrm{FFE}} \times h_{\mathrm{FFE}}\right)\end{array}$ & $\begin{array}{l}90 \mathrm{~mm} \times 100 \mathrm{~mm} \\
\times 200 \mu \mathrm{m}\end{array}$ & $\begin{array}{l}25 \mathrm{~mm} \times 10 \mathrm{~mm} \\
\times 100 \mu \mathrm{m}\end{array}$ & $\begin{array}{l}20 \mathrm{~mm} \times 11.7 \mathrm{~mm} \\
\times 50 \mu \mathrm{m}\end{array}$ & $\begin{array}{l}15 \mathrm{~mm} \times 23 \mathrm{~mm} \\
\times 5.0 \mu \mathrm{m}\end{array}$ \\
\hline FFE profile $A_{\mathrm{FFE}}$ & $1.8 \times 10^{-5} \mathrm{~m}^{2}$ & $2.5 \times 10^{-6} \mathrm{~m}^{2}$ & $1.0 \times 10^{-6} \mathrm{~m}^{2}$ & $7.5 \times 10^{-8} \mathrm{~m}^{2}$ \\
\hline Geometry factor $G_{\mathrm{FFE}}$ & $2.8 \mathrm{~mm}^{-1}$ & $2.0 \mathrm{~mm}^{-1}$ & $5.9 \mathrm{~mm}^{-1}$ & $1.5 \times 10^{2} \mathrm{~mm}^{-1}$ \\
\hline Conductivity $\kappa_{\mathrm{BGE}}$ & $1.7 \mathrm{mS} \mathrm{cm}^{-1}$ & $3.6 \times 10^{-2} \mathrm{mS} \mathrm{cm}^{-1}$ & $6.4 \times 10^{-3} \mathrm{mS} \mathrm{cm}^{-1}$ & $2.6 \times 10^{-1} \mathrm{mS} \mathrm{cm}^{-1}$ \\
\hline Length $L_{\text {trans }}$ of transfer line & $38 \mathrm{~cm}$ & $20 \mathrm{~cm}$ & $3 \mathrm{~cm}$ & $30 \mathrm{~cm}$ \\
\hline Diameter $d_{\text {trans }}$ of transfer line & $\begin{array}{l}1 / 16 ”=0.0625 ” \\
=1.59 \mathrm{~mm}\end{array}$ & $75 \mu \mathrm{m}$ & $50 \mu \mathrm{m} \times 20 \mu \mathrm{m}^{\mathrm{a}}$ & $100 \mu \mathrm{m}$ \\
\hline Cross-section $A_{\text {trans }}$ of transfer line & $2.0 \times 10^{-6} \mathrm{~m}^{2}$ & $4.4 \times 10^{-9} \mathrm{~m}^{2}$ & $1.0 \times 10^{-9} \mathrm{~m}^{2}$ & $7.9 \times 10^{-9} \mathrm{~m}^{2}$ \\
\hline Geometry factor $G_{\text {trans }}$ & $1.9 \times 10^{5} \mathrm{~m}^{-1}$ & $4.5 \times 10^{7} \mathrm{~m}^{-1}$ & $3.0 \times 10^{7} \mathrm{~m}^{-1}$ & $3.8 \times 10^{7} \mathrm{~m}^{-1}$ \\
\hline ESI voltage $V_{\text {ESI }}$ & $5500 \mathrm{~V}$ & $3000 \mathrm{~V}$ & $1200 \mathrm{~V}^{\mathrm{b}}$ & $4000 \mathrm{~V}$ \\
\hline Descriptor $H_{\text {asym }}$ & $2.5 \times 10^{-1}$ & $4.8 \times 10^{-3}$ & $9.7 \times 10^{-4}$ & $3.1 \times 10^{-2}$ \\
\hline Descriptor $H_{\text {leak }}$ & $1.0 \times 10^{2}$ & $8.8 \times 10^{-3}$ & $2.9 \times 10^{-3}$ & $8.5 \times 10^{-2}$ \\
\hline
\end{tabular}

1. P. Hoffmann, U. Häusig, P. Schulze, D. Belder, Angew. Chem. Int. Ed. 2007, 46, 4913-4916, DOI: 10.1002/anie.200605152.

2. C. Benz, M. Boomhoff, J. Appun, C. Schneider, D. Belder, Angew. Chem. Int. Ed. 2015, 54, 2766-2770, DOI: 10.1002/anie.201409663.

3. A. Chartogne, U. R. Tjaden, J. Van der Greef, Rapid Commun. Mass Spectrom. 2000, 14, 1269-1274, DOI: 10.1002/10970231(20000730)14:14<1269::AID-RCM24>3.0.CO;2-F.

4. J. K. Park, C. D. M. Campos, P. Neuzil, L. Abelmann, R. M. Guijt, A. Manz, Lab on a Chip 2015, 15, 3495-3502, DOI: 10.1039/C5LC00523J. 